\section{Scratching that itch - elucidating the spinal cord injury which causes reflex "phantom" scratching in canine syringomyelia}

\section{Zoe R. Nalborczyk ${ }^{1}$, Angus K. McFadyen ${ }^{2}$, Jelena Jovanovik ${ }^{3}$, Anna Tauro $^{3}$, Colin J. Driver $^{3}$, Noel Fitzpatrick ${ }^{3}$, Susan P. Knower ${ }^{1}$, Clare Rusbridge ${ }^{1,3}$}

1 School of Veterinary Medicine, Faculty of Health \& Medical Sciences Daphne Jackson Road, Guildford, Surrey, GU2 7AL

akm-stats, Glasgow, Scotland, UK

3 Fitzpatrick Referrals, Halfway Lane, Eashing, Godalming GU7 2QQ, Surrey, UK

\section{AIMS OF STUDY}

Syringomyelia (SM) is characterised by fluid filled cavities in the spinal cord. A classic sign of severe SM is a tendency to scratch towards one shoulder referred to as "phantom scratching". Stimulation of neck skin induces a rhythmic scratching action of the ipsilateral limb. Although easy to describe, the mechanism behind this action is less easy to elucidate. A popular explanation is that the dogs experience alloknesis (itch evoked by lightly touching the surrounding skin) or paraesthesia (a spontaneous or evoked sensation). However if affected dogs experience unusual sensations why do they make little or no skin contact? SM phantom scratching is similar to fictive scratch which develops a few months after transection of the caudal cervical spinal cord. In fictive scratch, stimulation of a skin receptive field results in a reflex scratching action due to hyperactivity of the scratching central pattern generator (CPG) i.e. neural circuits controlling a stereotyped sequence of muscle contractions. The similarity to fictive scratch suggests commonality of neural pathways. In this project we investigate the neuroanatomical site that relates to phantom scratching. We first investigate the hypothesis that phenomenon of phantom scratching is associated with a large dorsolateral syrinx in the upper cervical spinal cord segments. We then looked for an association to damage in other areas of the cervical spinal cord and investigated the hypothesis that phantom scratching is not just associated with a dorsolateral syrinx but one that extends to the superficial dorsal horn (SDH).

\section{METHODS}

Medical records from a two year period were searched for Cavalier King Charles spaniels that had magnetic resonance imaging and diagnosis of clinical SM. The cohort was divided into SM with phantom scratching (19 dogs) and SM but no phantom scratching (18 dogs). The MRI studies were anonymised, randomised and viewed in EFILM TM. For each transverse image the maximum perpendicular dimensions of the syrinx in each spinal cord quadrant was determined. Visual assessment was made as to whether the syrinx extended to the SDH.

\section{RESULTS}

The study found that phantom scratching is associated with a large dorsolateral syrinx that extends to the SDH in the C3-C6 spinal segments (C2-C5 vertebrae). The study did not find an association to damage of other areas of cervical spinal cord.

\section{CONCLUSION}

Phantom scratching in the dog is associated with a large syrinx that extends to the SDH in the C3-C6 spinal segments. We propose that phantom scratching is due to damage to projection neurons in lamina I of the $\mathrm{SDH}$ with consequent reduced descending inhibition to the lumbosacral scratching CPG. Drugs affecting SDH targets may be useful for management of phantom scratching. If a dog has SM extending to the SDH then it is at risk for phantom scratching. If an itchy SM affected dog has no SDH involvement then alternative explanations for scratching (e.g. allergic skin disease) should be investigated.

\section{FUNDING}

This project was funded by a 40th anniversary Petsavers Student Research Grant. The postgraduate research fees for SPK are funded by Cavalier Matters.

\section{Forebrain conformation} changes in Chiari-like malformation

\section{Chloe L. Cross' ${ }^{1}$, Angus K. McFadyen ${ }^{2}$, Jelena Jovanovik ${ }^{3}$, Anna Tauro ${ }^{3}$, Colin J. Driver $^{3}$, Noel Fitzpatrick ${ }^{3}$, Susan P. Knower ${ }^{1}$, Clare Rusbridge ${ }^{1,3}$}

1 School of Veterinary Medicine, Faculty of Health \& Medical Sciences Daphne Jackson Road, Guildford, Surrey GU2 7AL, Surrey, UK Akm-stats, Glasgow, Scotland, United Kingdom

3 Fitzpatrick Referrals, Halfway Lane, Eashing, Godalming GU7 2QQ, Surrey, UK

\begin{abstract}
AIMS OF STUDY
Chiari-like Malformation (CM) is a caudal fossa and craniocervical junction disorder resulting in cerebrospinal fluid pathway obstruction and variable syringomyelia (SM). Recent studies suggest that conformational changes are not confined to the hindbrain and that the entire skull base is foreshortened. Insufficient room for the forebrain may contribute to caudal displacement and overcrowding of the hindbrain. The olfactory bulbs $(\mathrm{OB})$ are ventrally orientated in brachycephalic dogs; it has been suggested that this may be more extreme in CM. Recently genetic studies have suggested a candidate gene Sall-1 for canine CM. Sall-1 deficiency in mice is associated with decreased OB size and defects in the human orthologue can be associated with skull abnormalities. We hypothesise cavalier King Charles
\end{abstract}

